\title{
Diabetic Management and Emotional Intelligence-An Emerging Direction in Current Research
}

\author{
Leehu Zysberg* \\ Associate Professor of Psychology, Gordon College of Education, Haifa Israel
}

Received: April 04, 2016; Accepted: April 27, 2016; Published: April 30, 2016

*Corresponding author: Leehu Zysberg, Associate Professor of Psychology, Gordon College of Education, Haifa Israel, Tel: +972-58-4877771; E-mail: leehuzysberg@yahoo.com; leehu@Gordon.academy

\begin{abstract}
Diabetes is a chronic condition affecting on average about $9 \%$ of the population, mostly type II with about. $4 \%$ diagnosed with type I diabetes mellitus. The condition poses multiple challenges to patients who struggle daily with the burden of monitoring, care, restrictive lifestyle and the stress inherent to the health and life threatening nature of the condition. While there is evidence in the existing literature supporting the pivotal role of emotions and emotion regulation in diabetic management, the evidence lacks in organization and conceptualization. The relatively new concept of emotional intelligence holds promise of providing an organizing framework for our understanding of the ways in which emotions are involved in determining diabetic management outcomes. A literature review of empirical studies focusing on emotional aspects and factors associated with diabetic management was conducted as a basis for suggesting a few directions in which emotional intelligence associates with diabetic outcomes, and propose directions for future research that hold promise to broaden our horizons in this venue.
\end{abstract}

\section{Introduction}

Diabetes Mellitus is a chronic, life threatening metabolic condition of a cumulative, increasingly destructive nature, manifested in multisystem failures, inflicting suffering, stress, decreased quality of life and eventually disability and death [1]. Diabetes' case prevalence ranges $2-21 \%$ in adult populations around the globe, and varies dramatically across cultures, socioeconomic class and gender. Among younger populations Type I diabetes prevalence ranges $0.2-2 \%$ depending on the above factors [2]. The existing evidence suggests that the conditions' prevalence will increase by $50-100 \%$ in the next 20 years [1], making it a modern day epidemic, taking its toll on individuals, their families, support systems and of course a huge burden on health systems, employment organizations and other systems.

The condition, once diagnosed, requires adherence to a strict, restrictive life style (e.g.: diet, exercise, etc.), and a painful monitoring and treatment regimen (e.g.: frequent blood tests, insulin injections, etc.). Given this reality, the literature has long examined the emotional and social implications of living with diabetes $[3,4]$. The evidence gathered across more than 40 years of research suggests that emotional responses and experience may play a role in the condition's onset, course, treatment effectiveness and outcomes [3]. In this paper I focus on the emotional aspects of the condition's management, often involving frustration, enduring pain and discomfort, conflict and ongoing stress, that are said to both increase physical susceptibility to complications as well as hinder appropriate and effective monitoring, care and adherence to treatment plans [4,5].

Despite the above evidence there is very little research exploring the role of emotional regulation, and emotional abilities in living with diabetes. Most of the existing research focuses on physiological paths associating emotional response patterns and physiological processes related to onset and exacerbation of the condition [6]. However, the possibility that managing one's and others' emotions in an effective manner can be pivotal in managing such a complex condition has not been properly addressed, until recently.

A relatively new concept emerging from research in the fields of organizational psychology, education and health science may prove to be relevant to our understanding of some of the mechanisms underlying the emotion-care effectiveness association [7]. Emotional Intelligence (EI) is a concept describing human potentials allowing individuals to identify emotions, integrate emotions in reasoning processes, perceive emotionally complex situations and experiences and as a result - effectively regulate emotional responses [8]. Preliminary evidence suggests EI positively associates with various indicators of health, functional status and general wellbeing $[9,10]$. The evidence however remains sporadic and lacking in an organizing framework, especially when it comes to chronic health conditions.

Given the preliminary evidence mentioned above, and the need to identify key factors underlying emotional processes in diabetic management, can the concept of EI account for variance in diabetes care outcomes and if so what insights can we draw from the current state of the research? The fragmental evidence in existence may support such a possibility [11].

To gain preliminary understanding of this new direction in our understanding of diabetic management the recent literature 
was reviewed to identify relevant evidence that may propose a model to guide future reasoning, theorizing and research into effective diabetic management.

\section{Methodology}

The literature was searched using PubMed and Google Scholar search-engines, using the keywords 'emotional intelligence' 'diabetes mellitus', 'type $1 /$ type 2 diabetes' and 'diabetic management' ('diabetes management' was not included since the term represents organizational and care related aspects that are less relevant here, as this paper focuses the individual level of care). The search was limited to the years 2000-2016, to stay aligned with the chronological timeline of the development of the empirical literature around EI. Non-empirical results were omitted, as did double results or opinion papers.

The purpose of the search was not to provide an in-depth and exhaustive review of the literature but rather to identify the key publications examining the above association and its nature. After omitting irrelevant content, non-empirical papers and doubles, only 8 studies were identified for the purpose of this brief paper.

An integrative critical reading of the sources is briefly summarized next, as the basis for a few insights and trends that may offer an added value for researchers interested in the emotional factors associated with the effective management of diabetes.

\section{A Brief Summary of Emerging Trends}

\section{Preliminary evidence to the association of EI and glycemic management}

Unpublished research offers indications [12], suggesting that positive associations may exist between self-report measures of EI and various outcome measures among diabetic patients (both self-report an objective such as blood sugar levels and HA1c). This is the earliest evidence regarding this possibility. For a long period this single study remained the only one in its field.

\section{Causal associations between EI and glycemic management outcomes}

Two studies conducted almost 9 years after the above data was first presented, tried to take the study of this association further by utilizing interventions to raise EI- related skills among intervention groups of type 2 diabetic patients, showing significant changes in both subjective measures and physiological measures of effective diabetic management. Physical indicators included both blood sugar levels as well as HA1c, an indicator of long term glycemic management. Though the study design was such that supported the hypothesis that EI does play a role in how effectively patients cope with the tasks of monitoring and caring for their condition, the sample sizes were small and the results could hardly be generalized $[13,14]$. These studies also failed to control for background variables that may intervene with the association.

\section{Clarifying the association in context}

As an answer to the issues raised above, recent studies examined the EI-diabetic outcomes association from a more sophisticated point of view: Acknowledging EI is not the only factor associated with emotional aspects of care and coping, thus examining EI alongside additional factors such as social support, personality traits associated with effective coping, cognitive abilities (associated with more effective problem solving skills) and so on. The studies' results showed a consistent pattern in which patients with higher EI managed their condition more effectively even after controlling for potential intervening factors (such as the ones mentioned above) [15-17]. As in the studies above, measures used included both blood sugar levels and HA1c as an indicator of long term balance. Larger samples sizes, in varying contexts (i.e.: inpatient clinics, hospitalization wards and home care settings included) add to the generalizability of the findings.

\section{The association in a broader context ?}

New, sporadic evidence also suggests that beyond the individual level, an environment characterized by higher levels of EI may have a buffer effect on the individuals coping with the challenges of their condition. Thus one recent study found that parents' EI levels positively associated with their diabetic children's care outcomes [16]. Another preliminary result suggests that the levels of EI among a small sample of adult diabetics' spouses associated favorably with their care outcomes, even after controlling for background variables and the patients' own levels of EI partly reported in [17]. In a recent review of the literature on the support of families of children with type 2 diabetes, though not always directly related to the concept of EI, evidence quoted supported associations between family caregivers' emotional abilities and coping skills and their children's diabetic management outcome indicators [18].

\section{A Brief Discussion and Directions for Future Research}

The research in this line of investigation is still in its infancy. The little evidence that has accumulated so far seems to support the possibility of a positive association between EI levels in individuals and their significant others and better glycemic control in both type 1 and type 2 DM patients. Sporadic evidence also suggests the association between the two is directional, maybe even causal. What may underlie this association though? How is EI linked with glycemic outcomes?

Evidence from adjacent fields e.g.: EI and eating disorders, see [19] suggests the association is not a direct one, but rather a mediated system in which EI provides the potential and personal resources that allow more efficient coping and handling of the challenges presented by the diagnosis. What are the mediating factors? The literature identifies a few strong 'candidates' that seem relevant within the context of diabetes monitoring and care, chief among them are: Perceived control, self-efficacy, stress regulation and management, and conflict management and resolution $[18,19]$. All these have been explored in psychological research and identified as underlying mechanisms accounting for a broad range of performance and behavior change. The little evidence in existence in this specific context suggests they may underlie effective glycemic control as well. 
Future research may focus on such potential mediation models to identify the main mechanisms EI enables and empowers that guide health promoting behaviors in this specific context. Should future research support the mediation model, we may gain new insight into additional paths for early identification and possible interventions with patients who may be at high risk of poor glycemic control, beyond education and instruction. Emotions are primary responses anchored at the most rudimentary levels of our physiology, perception and reactions to our world. Better understanding of these dynamics may provide a powerful tool for improving diabetic patients' quality of life and care.

\section{References}

1. Guariguata L, Whiting DR, Hambleton I, Beagley J, Linnenkamp U, Shaw JE. Global estimates of diabetes prevalence for 2013 and projections for 2035. Diabetes Res Clin Pract. 2014;103(2):137-49. doi: 10.1016/j.diabres.2013.11.002.

2. Pettitt DJ, Talton J, Dabelea D, Divers J, Imperatore G, Lawrence JM, et al. Prevalence of diabetes in US youth in 2009: the SEARCH for diabetes in youth study. Diabetes care. 2014;37(2):402-8. doi: 10.2337/dc131838.

3. Treuting TF. The role of emotional factors in the etiology and course of diabetes mellitus: a review of the recent literature. Am J Med Sci. 1962;244:93-109.

4. Close H, Davies AG, Price DA, Goodyer IM. Emotional difficulties in diabetes mellitus. Archives of disease in childhood. 1986;61(4):33740 .

5. Hartmann M, Kopf S, Kircher C, Faude-Lang V, Djuric Z, Augstein $\mathrm{F}$, et al. Sustained effects of a mindfulness-based stress-reduction intervention in type 2 diabetic patients design and first results of a randomized controlled trial (the Heidelberger diabetes and stressstudy). Diabetes care. 2012;35(5):945-7. doi: 10.2337/dc11-1343.

6. Novak M, Björck L, Giang KW, Heden-Ståhl C, Wilhelmsen L, Rosengren A. Perceived stress and incidence of Type 2 diabetes: a 35year follow-up study of middle-aged Swedish men. Diabetic medicine. 2013;30(1):e8-16. doi: 10.1111/dme.12037.

7. Con S, Parker JD, Saklofske DH. Emotional intelligence and physical health. In: Assessing emotional intelligence. US: Springer; 2009. p. 191-218.

8. Codier E, Muneno L, Franey K, Matsuura F. Is emotional intelligence an important concept for nursing practice? J Psychiatr Ment Health Nurs. 2010;17(10):940-8.

9. Schutte NS, Malouff JM, Thorsteinsson EB, Bhullar N, Rooke SE. A meta-analytic investigation of the relationship between emotional intelligence and health. Personality and Individual Differences. 2007;30;42(6):921-33.

10. Austin EJ, Saklofske DH, Egan V. Personality, well-being and health correlates of trait emotional intelligence. Personality and Individual differences. 2005;38(3):547-58.

11. Fisher EB, Thorpe CT, DeVellis BM, DeVellis RF. Healthy Coping, Negative Emotions, and Diabetes Management A Systematic Review and Appraisal. The Diabetes Educator. 2007;33(6):1080-103.

12.Samar, AD The relationship among emotional intelligence, self -management and glycemic control in individuals with type 1 diabetes (January 1, 2001). Doctoral Dissertations Available from Proquest. Paper AAI3012180.

13. Yalcin BM, Karahan TF, Ozcelik M, Igde FA. The effects of an emotional intelligence program on the quality of life and well-being of patients with type 2 diabetes mellitus. Diabetes Educ. 2008;34(6):1013-24. doi: $10.1177 / 0145721708327303$.

14. Karahan TF, YALÇIN BM. The effects of an emotional intelligence skills training program on anxiety, burnout and glycemic control in type 2 diabetes mellitus patients. Turkiye Klinikleri Journal of Medical Sciences. 2009;29(1):16-24.

15. Housiaux M, Luminet O, Van Broeck N, Dorchy H. Alexithymia is associated with glycaemic control of children with type 1 diabetes. Diabetes Metab. 2010;36(6 Pt 1):455-62. doi: 10.1016/j. diabet.2010.06.004.

16.Zysberg L, Lang T, Zisberg A. Parents' emotional intelligence and children's type I diabetes management. Journal of health psychology. 2013;18(9):1121-8.

17.Zysberg L, Bar YT, Goldman M. Emotional intelligence and glycemic management among type I diabetes patients. Journal of health psychology. 2015.

18. Zysberg L, Lang T. Supporting parents of children with type 1 diabetes mellitus: a literature review. Patient Intelligence. 2015;7:21-31.

19.Zysberg L, Tell E. Emotional Intelligence, Perceived Control, and Eating Disorders. SAGE Open. 2013;3(3):2158244013500285. 